\title{
VIT Regimen
}

National Cancer Institute

\section{Source}

National Cancer Institute. VIT Regimen. NCI Thesaurus. Code C148285.

A regimen consisting of vincristine, irinotecan and temozolomide that can be used for the treatment of Ewing sarcoma. 